Proyecciones Journal of Mathematics

Vol. 37, No 2, pp. 305-315, June 2018.

Universidad Católica del Norte

Antofagasta - Chile

\title{
Bound on H3(1) Hankel determinant for pre-starlike functions of order $\alpha$
}

\author{
D. Vamshee Krishna \\ GITAM University, India \\ and \\ D. Shalini
}

Sri Venkateswara College of Engineering and Technology, India

Received: September 2017. Accepted : March 2018

\begin{abstract}
The objective of this paper is to obtain best possible upper bound to the third Hankel determinant for the pre-starlike functions of order $\alpha(0 \leq \alpha<1)$, using Toeplitz determinants.
\end{abstract}

Keywords and phrases : Analytic function, pre-starlike function, convex function, upper bound, second and third Hankel functionals, positive real function, convolution, Toeplitz determinants.

2010 Mathematics Subject Classification : 30C45; 30C50. 


\section{Introduction}

Let $A$ denote the class of functions $f$ of the form

$$
f(z)=z+\sum_{n=2}^{\infty} a_{n} z^{n}
$$

in the open unit disc $E=\{z:|z|<1\}$. Let $S$ be the subclass of $A$ consisting of univalent functions. In 1985, Louis de Branges de Bourcia proved the Bieberbach conjecture, i.e., for a univalent function its $n^{\text {th }}$ Taylor coefficient is bounded by $n$ (see [3]). The bounds for the coefficients give information about the geometric properties of these functions. In particular, the growth and distortion properties of a normalized univalent function are determined by the bound of its second coefficient. Pommerenke [9] defined the following Hankel determinant of the function $f$ for $q \geq 1$ and $n \geq 1$, which has been extensively studied

$$
H_{q}(n)=\left|\begin{array}{cccc}
a_{n} & a_{n+1} & \cdots & a_{n+q-1} \\
a_{n+1} & a_{n+2} & \cdots & a_{n+q} \\
\vdots & \vdots & \vdots & \vdots \\
a_{n+q-1} & a_{n+q} & \cdots & a_{n+2 q-2}
\end{array}\right|\left(a_{1}=1\right) .
$$

It is easy to observe that $H_{2}(1)$ is the Fekete-Szegö functional. Fekete and Szegö then further generalized the estimate $\left|a_{3}-\mu a_{2}^{2}\right|$ with $\mu$ real and $f \in S$. Ali [1] found sharp bounds on the first four coefficients and sharp estimate for the Fekete-Szegö functional $\left|\gamma_{3}-t \gamma_{2}^{2}\right|$, where $t$ is real, for the inverse function of $f$ defined as $f^{-1}(w)=w+\sum_{n=2}^{\infty} \gamma_{n} w^{n}$, when $f \in \widetilde{S T}(\alpha)$, the class of strongly starlike functions of order $\alpha(0<\alpha \leq 1)$. Further, sharp bounds for the second Hankel determinant functional

$$
H_{2}(2)=\left|\begin{array}{ll}
a_{2} & a_{3} \\
a_{3} & a_{4}
\end{array}\right|=a_{2} a_{4}-a_{3}^{2} \text {, when } q=2 \text { and } n=2,
$$

were obtained for various subclasses of univalent and multivalent analytic functions. In particular, Janteng et al. [6] obtained sharp bound to the functional $\left|H_{2}(2)\right|$ for the well known subclasses of $S$, namely, starlike and convex functions denoted by $S^{*}$ and $\mathcal{K}$ respectively and showed that $\left|a_{2} a_{4}-a_{3}^{2}\right| \leq 1$ and $\left|a_{2} a_{4}-a_{3}^{2}\right| \leq \frac{1}{8}$ respectively. For our discussion, in this paper, we consider the Hankel determinant in the case of $q=3$ and $n=1$, denoted by $H_{3}(1)$, given by

$$
H_{3}(1)=\left|\begin{array}{lll}
a_{1} & a_{2} & a_{3} \\
a_{2} & a_{3} & a_{4} \\
a_{3} & a_{4} & a_{5}
\end{array}\right| .
$$


On expanding the determinant by means of third column and then applying the triangle inequality, we obtain

$$
\left|H_{3}(1)\right| \leq\left|a_{3}\right|\left|a_{2} a_{4}-a_{3}^{2}\right|+\left|a_{4}\right|\left|a_{2} a_{3}-a_{4}\right|+\left|a_{5}\right|\left|a_{3}-a_{2}^{2}\right| .
$$

Babalola [2] obtained sharp upper bounds to the functional $\left|a_{2} a_{3}-a_{4}\right|$ and $\left|H_{3}(1)\right|$ for the familiar subclasses namely starlike and convex functions respectively denoted by $S^{*}$ and $\mathcal{K}$ of $S$. The sharp upper bound to the second Hankel functional $\left|H_{2}(2)\right|=\left|a_{2} a_{4}-a_{3}^{2}\right|$ for these classes were obtained by Janteng et.al [6]. Motivated by the results obtained by different authors working in this direction, in this paper, using convolution technique, with the help of Toeplitz determinants, we seek an upper bound to the functional $\left|a_{2} a_{3}-a_{4}\right|$ and hence for $\left|H_{3}(1)\right|$, when the function $f$ given in (1.1) belongs to the class of pre-starlike functions of order $\alpha(0 \leq \alpha<1)$, defined as follows.

Definition 1.1. A function $f(z) \in A$ is said to be starlike function of order $\alpha(0 \leq \alpha<1)$, denoted by $f \in S^{*}(\alpha)$, if and only if

$$
\operatorname{Re}\left\{\frac{z f^{\prime}(z)}{f(z)}\right\}>\alpha, \quad z \in E .
$$

Definition 1.2. A function $f(z) \in A$ is said to be convex function of order $\alpha(0 \leq \alpha<1)$, denoted by $f \in \mathcal{K}(\alpha)$, if and only if

$$
\operatorname{Re}\left\{1+\frac{z f^{\prime \prime}(z)}{f^{\prime}(z)}\right\}>\alpha, \quad z \in E .
$$

From the Definitions 1.1 and 1.2, we observe that $f \in \mathcal{K}(\alpha) \Leftrightarrow z f^{\prime} \in$ $S^{*}(\alpha)$.

Definition 1.3. A function $f \in A$ is said to be in the class of pre-starlike functions of order $\alpha(0 \leq \alpha<1)$, denoted by $R_{\alpha}$, if and only if

$$
f(z) * s_{\alpha}(z) \in S^{*}(\alpha), \quad z \in E,
$$

where $*$ denotes the convolution of two analytic functions and $s_{\alpha}(z)=\frac{z}{(1-z)^{2(1-\alpha)}}$ is the extremal function for the class $S^{*}(\alpha)$.

The class $R_{\alpha}$ was introduced and studied by Ruscheweyh [10].

$$
\text { Take } c(\alpha, n)=\frac{\prod_{k=2}^{n}(k-2 \alpha)}{(n-1) !}, \quad \text { for } n=2,3, \ldots
$$


so that $s_{\alpha}(z)$ can be written in the form

$$
s_{\alpha}(z)=z+\sum_{n=2}^{\infty} c(\alpha, n) z^{n}
$$

noting that $c(\alpha, n)$ is a decreasing function of $\alpha$ with

$$
\lim _{n \rightarrow \infty} c(\alpha, n)=\left\{\begin{array}{l}
\infty, \text { if } \alpha<\frac{1}{2}, \\
1, \text { if } \alpha=\frac{1}{2}, \\
0, \text { if } \alpha>\frac{1}{2} .
\end{array}\right.
$$

Ruscheweyh (see [11]) also showed that the necessary and sufficient condition for $f \in R_{\alpha}$ is that the function

$$
G(\alpha, z)=\frac{f(z) * \frac{s_{\alpha}(z)}{(1-z)}}{f(z) * s_{\alpha}(z)},
$$

satisfy $\operatorname{Re} G(\alpha, z)>\frac{1}{2}, z \in E$. Since $s_{1}(z)=z$, we say that $f$ is pre-starlike function of order 1 , if and only if

$$
\operatorname{Re} \frac{f(z)}{z}>\frac{1}{2}, \quad z \in E .
$$

Note that $R_{0}=\mathcal{K}$ and $R_{\frac{1}{2}}=S^{*}\left(\frac{1}{2}\right)$.

It was shown that $R_{\alpha} \subset R_{\beta}$, for $0 \leq \alpha<\beta \leq 1$, which generalizes the well-known result that $\mathcal{K}(0) \subset S^{*}\left(\frac{1}{2}\right)$.

In proving our results in section 3, we require the following Lemmas:

\section{Preliminaries}

Let $P$ denote the class of functions consisting of $p$, such that

$$
p(z)=1+\sum_{n=1}^{\infty} c_{n} z^{n},
$$

which are analytic (regular) in the open unit disc $E$ and satisfy $\operatorname{Re} p(z)>0$ for any $z \in E$. Here $p(z)$ is called a Caratheòdory function [4].

Lemma 2.1. ([8, 12]) If $p \in P$, then $\left|c_{k}\right| \leq 2$, for each $k \geq 1$ and the extremal function in this case is $\frac{1+z}{1-z}$. 
Lemma 2.2. Let $p \in P$, then

$$
2 c_{2}=c_{1}^{2}+y\left(4-c_{1}^{2}\right)
$$

and

$$
4 c_{3}=\left\{c_{1}^{3}+2 c_{1}\left(4-c_{1}^{2}\right) y-c_{1}\left(4-c_{1}^{2}\right) y^{2}+2\left(4-c_{1}^{2}\right)\left(1-|y|^{2}\right) \zeta\right\},
$$

for some $y, \zeta$ such that $|y| \leq 1$ and $|\zeta| \leq 1$.

We apply the classical method initiated by Libera and Zlotkiewicz [7], which has been used widely, in obtaining our results.

\section{Main Results}

Theorem 3.1. If $f \in R_{\alpha}$ then

$$
\left|a_{2} a_{3}-a_{4}\right| \leq \frac{4}{3 \sqrt{3}(3-2 \alpha)}, \text { for } \quad(0 \leq \alpha<1) .
$$

Proof. Let $f(z)=z+\sum_{n=2}^{\infty} a_{n} z^{n} \in R_{\alpha}$, by virtue of Definition 1.3, we have

$$
f(z) * s_{\alpha}(z) \in S^{*}(\alpha), \quad \forall z \in E .
$$

By the convolution, we obtain

$$
\begin{gathered}
g(z)=f(z) * s_{\alpha}(z)=\left\{z+\sum_{n=2}^{\infty} a_{n} z^{n}\right\} *\left\{z+\sum_{n=2}^{\infty} c(\alpha, n) z^{n}\right\} \\
z+\sum_{n=2}^{\infty} c(\alpha, n) a_{n} z^{n} .
\end{gathered}
$$

Since $g(z) \in S^{*}(\alpha)$, from Definition 1.1, there exists an analytic function $p \in P$ in the open unit disc $E$ with $p(0)=1$ and $\operatorname{Re} p(z)>0$ such that

$$
\frac{z g^{\prime}(z)-\alpha g(z)}{(1-\alpha) g(z)}=p(z) \Leftrightarrow z g^{\prime}(z)-\alpha g(z)=(1-\alpha) g(z) p(z) .
$$

Using the series representations for $g(z)$ and $p(z)$ in (3.3), we have

$$
\begin{aligned}
& z\left\{1+\sum_{n=2}^{\infty} c(\alpha, n) n a_{n} z^{n-1}\right\}-\alpha\left\{z+\sum_{n=2}^{\infty} c(\alpha, n) a_{n} z^{n}\right\} \\
& =(1-\alpha)\left\{z+\sum_{n=2}^{\infty} c(\alpha, n) a_{n} z^{n}\right\}\left\{1+\sum_{n=1}^{\infty} c_{n} z^{n}\right\} .
\end{aligned}
$$


Upon simplification, we obtain

$$
\begin{aligned}
& c(2, \alpha) a_{2}+2 c(3, \alpha) a_{3} z+3 c(4, \alpha) a_{4} z^{2}+4 c(5, \alpha) a_{5} z^{3}+\ldots= \\
& (1-\alpha) \times\left[c_{1}+\left\{c_{2}+c(2, \alpha) c_{1} a_{2}\right\} z+\left\{c_{3}+c(2, \alpha) c_{2} a_{2}+c(3, \alpha) c_{1} a_{3}\right\} z^{2}+\right. \\
& \left.\left\{c_{4}+c(2, \alpha) c_{3} a_{2}+c(3, \alpha) c_{2} a_{3}+c(4, \alpha) c_{1} a_{4}\right\} z^{3}+\ldots\right] .
\end{aligned}
$$

Equating the coefficients of $z^{0}, z, z^{2}$ and $z^{3}$ respectively in (3.4), after simplifying, we get

$$
\begin{aligned}
& a_{2}=\frac{c_{1}}{2} ; \quad a_{3}=\frac{1}{2(3-2 \alpha)}\left\{c_{2}+(1-\alpha) c_{1}^{2}\right\} \\
& a_{4}=\frac{1}{4(2-\alpha)(3-2 \alpha)}\left\{2 c_{3}+3(1-\alpha) c_{1} c_{2}+(1-\alpha)^{2} c_{1}^{3}\right\} . \\
& a_{5}=\frac{1}{2(5-2 \alpha)(4-2 \alpha)(3-2 \alpha)} \times \\
& \left\{6 c_{4}+8(1-\alpha) c_{1} c_{3}+3(1-\alpha) c_{2}^{2}+6(1-\alpha)^{2} c_{1}^{2} c_{2}+(1-\alpha)^{3} c_{1}^{4}\right\} .
\end{aligned}
$$

Substituting the values of $a_{2}, a_{3}$ and $a_{4}$ from (3.5) in the functional $\left|a_{2} a_{3}-a_{4}\right|$, which simplifies to give

$$
\left|a_{2} a_{3}-a_{4}\right|=\frac{1}{4(2-\alpha)(3-2 \alpha)}\left|-2 c_{3}+(-1+2 \alpha) c_{1} c_{2}+(1-\alpha) c_{1}^{3}\right| .
$$

The above expression is equivalent to

$$
\left|a_{2} a_{3}-a_{4}\right|=\frac{1}{4(2-\alpha)(3-2 \alpha)}\left|d_{1} c_{3}+d_{2} c_{1} c_{2}+d_{3} c_{1}^{3}\right|,
$$

$$
\text { where } d_{1}=-2 ; \quad d_{2}=-1+2 \alpha ; \quad d_{3}=1-\alpha .
$$

Using the values of $c_{2}$ and $c_{3}$ from (2.2) and (2.3) respectively from Lemma 2.2 on the right-hand side of (3.6), we have

$$
\begin{aligned}
& \left|d_{1} c_{3}+d_{2} c_{1} c_{2}+d_{3} c_{1}^{3}\right|=\mid d_{1} \times \frac{1}{4}\left\{c_{1}^{3}+2 c_{1}\left(4-c_{1}^{2}\right) y-c_{1}\left(4-c_{1}^{2}\right) y^{2}\right. \\
& \left.+2\left(4-c_{1}^{2}\right)\left(1-|y|^{2}\right) \zeta\right\}+d_{2} c_{1} \times \frac{1}{2}\left\{c_{1}^{2}+y\left(4-c_{1}^{2}\right)\right\}+d_{3} c_{1}^{3} \mid .
\end{aligned}
$$

Applying the triangle inequality and the fact $|\zeta| \leq 1$ in the expression (3.8), which simplifies to 


$$
\begin{aligned}
& 4\left|d_{1} c_{3}+d_{2} c_{1} c_{2}+d_{3} c_{1}^{3}\right| \leq \mid\left(d_{1}+2 d_{2}+4 d_{3}\right) c_{1}^{3}+2 d_{1}\left(4-c_{1}^{2}\right)+ \\
& 2\left(d_{1}+d_{2}\right) c_{1}\left(4-c_{1}^{2}\right)|y|-d_{1}\left(c_{1}+2\right)\left(4-c_{1}^{2}\right)|y|^{2} \mid .
\end{aligned}
$$

From (3.7), we can now write

$$
d_{1}+2 d_{2}+4 d_{3}=0 ; \quad d_{1}+d_{2}=-3+2 \alpha .
$$

From the expressions (3.9) and (3.10), we have

$4\left|d_{1} c_{3}+d_{2} c_{1} c_{2}+d_{3} c_{1}^{3}\right| \leq\left.\left|4\left(4-c_{1}^{2}\right)+2(-3+2 \alpha) c_{1}\left(4-c_{1}^{2}\right)\right| y\left|+2\left(c_{1}+2\right)\left(4-c_{1}^{2}\right)\right| y\right|^{2} \mid$.

Since $c_{1}=c \in[0,2]$, noting that $c_{1}+a \geq c_{1}-a$, where $a \geq 0$, applying the triangle inequality and replacing $|y|$ by $\mu$ on the right-hand side of the above inequality, we get

$$
\begin{aligned}
& 2\left|d_{1} c_{3}+d_{2} c_{1} c_{2}+d_{3} c_{1}^{3}\right| \leq\left[2+(3-2 \alpha) c \mu+(c-2) \mu^{2}\right]\left(4-c^{2}\right) \\
& =F(c, \mu), \text { for } 0 \leq \mu=|y| \leq 1,
\end{aligned}
$$

where

$$
F(c, \mu)=\left[2+(3-2 \alpha) c \mu+(c-2) \mu^{2}\right]\left(4-c^{2}\right) .
$$

Further, we maximize the function $F(c, \mu)$ on the closed region $[0,2] \times$ $[0,1]$. Differentiating $F(c, \mu)$ partially with respect to $\mu$ and $c$ respectively, we obtain

$$
\begin{gathered}
\frac{\partial F}{\partial \mu}=[(3-2 \alpha) c+(c-2) \mu]\left(4-c^{2}\right) \\
\text { and } \frac{\partial F}{\partial c}=-4 c+(3-2 \alpha)\left(4-3 c^{2}\right) \mu+\left(4+4 c-3 c^{2}\right) \mu^{2} .
\end{gathered}
$$

For the extreme values of $F(c, \mu)$, consider

$$
\frac{\partial F}{\partial \mu}=0 \quad \text { and } \quad \frac{\partial F}{\partial c}=0 .
$$


In view of (3.16), on solving the equations in (3.14) and (3.15), we obtain the critical point for the function $F(c, \mu)$ which lies in the closed region $[0,2] \times[0,1]$ is $(0,0)$ only. At the critical point $(0,0)$, we observe that

$$
\frac{\partial^{2} F}{\partial \mu^{2}}=-4<0 ; \quad \frac{\partial^{2} F}{\partial c^{2}}=-16<0 ; \quad \frac{\partial^{2} F}{\partial c \partial \mu}=4(3-2 \alpha)
$$

and

$$
\left[\left(\frac{\partial^{2} F}{\partial \mu^{2}}\right)\left(\frac{\partial^{2} F}{\partial c^{2}}\right)-\left(\frac{\partial^{2} F}{\partial c \partial \mu}\right)^{2}\right]=16\left(-4 \alpha^{2}+12 \alpha-5\right)<0, \text { for } 0 \leq \alpha<1
$$

Therefore, at the point $(0,0)$, the function $F(c, \mu)$ has local minimum. Now, we search for the critical points on the boundary of the region $[0,2] \times$ $[0,1]$. (i) At $L_{1}=\{(2, \mu) / 0 \leq \mu \leq 1\}$, we have $F(2, \mu)=0$, which is a constant.

(ii) At $L_{2}=\{(0, \mu) / 0 \leq \mu \leq 1\}$, we obtain $F(2, \mu)=8-8 \mu^{2}$, which gives us the same critical point $(0,0)$. (iii) At $L_{3}=\{(c, 0) / 0 \leq c \leq 2\}$, we get $F(c, 0)=8-2 c^{2}$, which also gives us the same critical point $(0,0)$. (iv) At $L_{4}=\{(c, 1) / 0 \leq c \leq 2\}$, we get $F(c, 1)=8(2-\alpha)-2(2-\alpha) c^{3}$.

$$
\begin{gathered}
\text { Let } G(c)=F(c, 1)=8(2-\alpha)-2(2-\alpha) c^{3}, \\
G^{\prime}(c)=8(2-\alpha)-6(2-\alpha) c^{2} \\
G^{\prime \prime}(c)=-12(2-\alpha) c .
\end{gathered}
$$

For optimum value of $G(c)$, consider $G^{\prime}(c)=0$. From (3.18), we get $c^{2}=\frac{4}{3}$. Since $c \in[0,2]$, consider $c=\frac{2}{\sqrt{3}}$ only. Substituting the value of $c=\frac{2}{\sqrt{3}}$ in (3.19), we observe that $G^{\prime \prime}(c)<0$, for $0 \leq \alpha<1$. Therefore, by the second derivative test, $G(c)$ has maximum value at $c=\frac{2}{\sqrt{3}}$, from (3.17), it is obtained to be

$$
G_{\max }=\frac{32(2-\alpha)}{3 \sqrt{3}} .
$$


Simplifying the expressions (3.6), (3.12) and (3.20), we get

$$
\left|a_{2} a_{3}-a_{4}\right| \leq \frac{4}{3 \sqrt{3}(3-2 \alpha)}
$$

This completes the proof of Theorem 3.1.

Remark 3.2. Choosing $\alpha=0$ in (3.21), we obtain $\left|a_{2} a_{3}-a_{4}\right| \leq \frac{4}{9 \sqrt{3}}$. This inequality is sharp and coincides with that of Babalola [2].

The following Theorem is a straightforward verification on applying Theorem 3.1.

Theorem 3.3. If $f \in R_{\alpha}$ then $\left|a_{3}-a_{2}^{2}\right| \leq \frac{1}{(3-2 \alpha)}$, for $(0 \leq \alpha<1)$.

Remark 3.4. If $\alpha=0$ in the above Theorem, we get $\left|a_{3}-a_{2}^{2}\right| \leq \frac{1}{3}$, this result is same as the result obtained by Babalola [2].

Theorem 3.5. If $f(z) \in R_{\alpha}$ then we have $\left|a_{k}\right| \leq 1$, for $k \geq 2$.

Proof. Using the fact that $\left|c_{n}\right| \leq 2$, for $n \in N=\{1,2,3 \cdots\}$, with the help of $c_{2}$ and $c_{3}$ values given in (2.2) and (2.3) respectively together with the values obtained in (3.5), we obtain $\left|a_{k}\right| \leq 1$, for $k \geq 2$. This completes the proof of our Theorem 3.5.

Substituting the results of Theorems 3.1, 3.3, 3.5 and the known result $\left|a_{2} a_{4}-a_{3}^{2}\right| \leq \frac{1}{8(1-\alpha)}$, for $\left(0 \leq \alpha<\frac{1}{2}\right)$ (see [14]) in the inequality given in (1.4), which simplifies to obtain the following Corollary.

Corollary 3.6. If $f(z) \in R_{\alpha}$, then

$$
\left|H_{3}(1)\right| \leq\left[\frac{32(1-\alpha)+\sqrt{3}(33-30 \alpha)}{24 \sqrt{3}(1-\alpha)(3-2 \alpha)}\right] .
$$

Remark 3.7. Choosing $\alpha=0$, the above corollary coincides with the result obtained by Babalola [2].

Acknowledgements : The authors thank Prof. T. RamReddy for useful discussion and suggestions. The authors also express their sincere thanks to the Editor and the esteemed Referees for their valuable suggestions to improve the manuscript. 


\section{References}

[1] R. M. Ali, Coefficients of the inverse of strongly starlike functions, Bull. Malays. Math. Sci. Soc., (2nd Series), 26 (1), pp. 63-71, (2003).

[2] K. O. Babalola, On $H_{3}(1)$ Hankel determinant for some classes of univalent functions, Inequality Theory and Applications, 6, pp. 1-7, (2010).

[3] L. de Branges de Bourcia, A proof of Bieberbach conjecture, Acta Mathematica, 154 (1-2), pp. 137-152, (1985).

[4] P. L. Duren, Univalent functions, Vol. 259 of Grundlehren der Mathematischen Wissenschaften, Springer, New York, USA, (1983).

[5] U. Grenander and G. Szegö, Toeplitz forms and their applications. 2nd ed. New York (NY): Chelsea Publishing Co., (1984).

[6] A. Janteng, S. A. Halim and M. Darus, Hankel Determinant for starlike and convex functions, Int. J. Math. Anal. (Ruse), 1 (13), pp. 619-625, (2007).

[7] R. J. Libera and E. J. Zlotkiewicz, Coefficient bounds for the inverse of a function with derivative in $P$, Proc. Amer. Math. Soc., 87 (2), pp. 251-257, (1983).

[8] Ch. Pommerenke, Univalent functions, Gottingen: Vandenhoeck and Ruprecht; (1975).

[9] Ch. Pommerenke, On the coefficients and Hankel determinants of univalent functions, J. Lond. Math. Soc., s1-41 (1), pp. 111-122, (1966).

[10] St. Ruscheweyh, Linear operators between classes of pre-starlike functions, Comm. Math. Helv., 52, pp. 497-509, (1977).

[11] H. Silverman and E. M. Silvia, Pre-starlike functions with negative coefficients, Int. J. Math. Math. Sci., 2 (3), pp. 427-439, (1979).

[12] B. Simon, Orthogonal polynomials on the unit circle, part 1. Classical theory. Vol. 54, American mathematical society colloquium publications. Providence (RI): American Mathematical Society; (2005). 
[13] D. Vamshee Krishna and T. RamReddy, Coefficient inequality for parabolic star like functions of order alpha, Afr. Mat., 27 (1-2), pp. 121-132, (2016).

[14] D. Vamshee Krishna and T. RamReddy, An upper bound to the second Hankel determinant for pre-star like functions of order $\alpha$, Le Matematiche, 70 (2), pp. 109-122, (2015).

[15] D. Vamshee Krishna and T. RamReddy, Coefficient inequality for certain $p$ - valent analytic functions, Rocky Mountain J. Math., 44 (6), pp. 1941-1959, (2014).

\author{
D. Vamshee Krishna \\ Department of Mathematics, \\ GITAM University \\ Visakhapatnam 530 045, A. P., \\ India \\ e-mail : vamsheekrishna1972gmail.com \\ and

\section{Shalini} \\ Department of Mathematics, \\ Sri Venkateswara College of Engineering and Technology, \\ Affiliated to JNTUK, \\ Etcherla 532 410, A. P., \\ India \\ e-mail : shaliniraj1005@gmail.com
}

